\title{
ON THE MAXIMUM MODULUS AND MAXIMUM TERM OF COMPOSITION OF ENTIRE FUNCTIONS
}

\author{
S. K. VAISH AND R. CHANKANYAL
}

\begin{abstract}
We study some growth properties of maximum modulus and maximum term of composition of entire functions of $(p, q)$-order as compared to the growth of their corresponding left and right factors. Some of the results that we obtain here generalize and improve the known results of Singh and Baloria, and, Song and Yang.
\end{abstract}

\section{Introduction}

Let $f(z)=\sum_{n=0}^{\infty} a_{n} z^{n}$ be an entire function. Then as usual $\mu(r, f)=\max _{n \geq 0}\left\{\left|a_{n}\right| r^{n}\right\}$ is called the maximum term of $f(z)$ on $|z|=r$ and $M(r, f)=\max _{|z|=r}|f(z)|$ is called the maximum modulus of $f(z)$ on $|z|=r$. The concept of $(p, q)$-order and lower $(p, q)$-order of $f(z)$ having index pair $(p, q),(p \geq q \geq 1, p \geq 2)$, was introduced by Juneja, Kapoor and Bajpai [2]. Thus $f(z)$ is said to be of $(p, q)$-order $\rho(f)$ and lower $(p, q)$-order $\lambda(f)$, if

$$
\lim _{r \rightarrow \infty} \sup _{\inf } \frac{\log ^{[p]} M(r, f)}{\log ^{[q]} r}=\begin{gathered}
\rho(f) \\
\lambda(f)
\end{gathered}, \quad b \leq \lambda(f) \leq \rho(f) \leq \infty
$$

where $b=1$ if $p=q$ and zero otherwise and $\exp ^{[0]} x=\log ^{[0]} x=x ; \exp ^{[m]} x=\log ^{[-m]} x=$ $\exp \left(\exp ^{[m-1]} x\right)=\log \left(\log { }^{[-m-1]} x\right) ; m= \pm 1, \pm 2, \ldots$ Throughout this paper whenever $\left(\log ^{[m]} x\right)^{\beta}(0<\beta<\infty)$ occurs, it is understood that $x$ is such that this expression is a real number.

\section{Known Results}

In this section we state some known results in the form of Lemmas which will be needed in the sequel.

Lemma 1. (Juneja, Kapoor and Bajpai [2]). Let $f(z)$ be an entire function of $(p, q)$-order $\rho(f)$ and lower $(p, q)$-order $\lambda(f)$, then

$$
\lim _{r \rightarrow \infty} \operatorname{sinf} \frac{\log ^{[p]} \mu(r, f)}{\log ^{[q]} r}=\begin{gathered}
\rho(f) \\
\lambda(f)
\end{gathered} .
$$

Received May 2, 2003.

2000 Mathematics Subject Classification. 30D35.

Key words and phrases. Entire function, composition, $(p, q)$-order, maximum modulus, maximum term. 
Lemma 2 (Clunie [1]). Let $f(z)$ and $g(z)$ be two entire functions with $g(0)=0$. Let $\alpha$ satisfy $0<\alpha<1$ and $c(\alpha)=(1-\alpha)^{2} /(4 \alpha)$. Then, for $r>0$,

$$
M(r, f \circ g) \geq M(c(\alpha) M(\alpha r, g), f)
$$

Further, if $g(z)$ is any entire function with $\alpha=1 / 2$, for sufficiently large values of $r$,

$$
M(r, f \circ g) \geq M\left(\frac{1}{8} M\left(\frac{r}{2}, g\right)-|g(0)|, f\right) .
$$

Lemma 3 (Singh [3]). Let $f(z)$ and $g(z)$ be two entire functions with $g(0)=0$. Let $\alpha$ satisfying $0<\alpha<1$ and $c(\alpha)=(1-\alpha)^{2} /(4 \alpha)$. Also, let $0<\delta<1$, then

$$
\mu(r, f \circ g) \geq(1-\delta) \mu(c(\alpha) \mu(\alpha \delta r, g), f)
$$

and, if $g(z)$ is any entire function, then with $\alpha=\delta=1 / 2$, for sufficiently large values of $r$,

$$
\mu(r, f \circ g) \geq \frac{1}{2} \mu\left(\frac{1}{8} \mu\left(\frac{r}{4}, g\right)-|g(0)|, f\right)
$$

In this paper we study some growth properties of maximum modulus and maximum term of composition of entire functions of $(p, q)$-order as compared to the growth of their corresponding left and right factors. Some of the results that we obtain here generalize and improve the known results of Singh and Baloria [4] and Song and Yang [5].

\section{Main Results}

Theorem 1. Let $f$ and $g$ be two entire functions such that $0<\lambda(f) \leq \rho(f)<\infty$ and $0<\lambda(g) \leq \rho(g)<\infty$. Then for every positive constant $\gamma, p>q$ and every real number $x$,

$$
\lim _{r \rightarrow \infty} \frac{\log ^{[p]} M(r, f o g)}{\left\{\log ^{[p]} M\left(r^{\gamma}, f\right)\right\}^{1+x}}=\infty
$$

and

$$
\lim _{r \rightarrow \infty} \frac{\log ^{[p]} M(r, f o g)}{\left\{\log ^{[p]} M\left(r^{\gamma}, g\right)\right\}^{1+x}}=\infty .
$$

Proof. If $x$ is such that $1+x \leq 0$, then the theorem is obvious. So, we suppose that $1+x>0$. Now, for all sufficiently large values of $r$, we get from (2.3)

$$
M(r, f \circ g) \geq M\left(\frac{1}{16} M\left(\frac{r}{2}, g\right), f\right) .
$$


This gives,

$$
\begin{aligned}
\log ^{[p]} M(r, f o g) & \geq \log ^{[p]} M\left(\frac{1}{16} M\left(\frac{r}{2}, g\right), f\right) \\
& >(\lambda(f)-\varepsilon) \log ^{[q]}\left(\frac{1}{16} M\left(\frac{r}{2}, g\right)\right) \\
& >(\lambda(f)-\varepsilon) \log ^{[q-1]}\left\{\log \frac{1}{16}+\exp ^{[p-2]}\left(\log ^{[q-1]}\left(\frac{r}{2}\right)\right)^{\lambda(g)-\varepsilon}\right\} \\
& =(\lambda(f)-\varepsilon) \exp ^{[p-q-1]}\left(\log ^{[q-1]}\left(\frac{r}{2}\right)\right)^{\lambda(g)-\varepsilon}+(\lambda(f)-\varepsilon) o(1)
\end{aligned}
$$

where we choose $0<\varepsilon<\min (\lambda(f), \lambda(g))$.

Also, for all $r \geq r_{0}$,

$$
\log ^{[p]} M(r, f)<(\rho(f)+\varepsilon) \log ^{[q]} r .
$$

Now, it is possible to choose $r$ sufficiently large so that $r^{\gamma} \geq r_{0}$. Thus

$$
\left\{\log ^{[p]} M\left(r^{\gamma}, f\right)\right\}^{1+x}<(\rho(f)+\varepsilon)^{1+x}\left\{\log ^{[q]}\left(r^{\gamma}\right)\right\}^{1+x} .
$$

From (3.3) and (3.4) it follows that for all sufficiently large values of $r$,

$$
\frac{\log ^{[p]} M(r, f o g)}{\left\{\log ^{[p]} M\left(r^{\gamma}, f\right)\right\}^{1+x}}>\frac{(\lambda(f)-\varepsilon) \exp ^{[p-q-1]}\left\{\log ^{[q-1]}\left(\frac{r}{2}\right)\right\}^{\lambda(g)-\varepsilon}+(\lambda(f)-\varepsilon) o(1)}{(\rho(f)+\varepsilon)^{1+x}\left\{\log ^{[q]}\left(r^{\gamma}\right)\right\}^{1+x}} .
$$

Since $\left\{\exp ^{[p-q-1]}\left(\log ^{[q-1]}(r / 2)\right)\right\}^{\lambda(g)-\varepsilon} /\left\{\log ^{[q]}\left(r^{\gamma}\right)\right\}^{1+x} \rightarrow \infty$ as $r \rightarrow \infty$, statement (3.1) follows from (3.5).

Statement (3.2) follows similarly by using the following inequality in plcae of (3.4),

$$
\left\{\log ^{[p]} M\left(r^{\gamma}, g\right)\right\}^{1+x}<(\rho(g)+\varepsilon)^{1+x}\left\{\log ^{[q]}\left(r^{\gamma}\right)\right\}^{1+x}
$$

for all sufficiently large values of $r$. This proves the theorem.

Note. Theorem 1 need not be true if either $\lambda(g)=0$ or $\lambda(f)=0$.

For example:

Let $g(z)=z, x=0$ and $\gamma=1$, then $\lambda(g)=0$ and we find

$$
\lim _{r \rightarrow \infty} \frac{\log ^{[p]} M(r, f o g)}{\left\{\log ^{[p]} M\left(r^{\gamma}, g\right)\right\}^{1+x}}=1 .
$$

Similarly, if $f(z)=z, x=0$ and $\gamma=1$, then $\lambda(f)=0$ and

$$
\lim _{r \rightarrow \infty} \frac{\log ^{[p]} M(r, \text { fog })}{\left\{\log ^{[p]} M\left(r^{\gamma}, g\right)\right\}^{1+x}}=1 .
$$




\section{Remark 1.}

(i) For $p=2, q=1$ and $x=0$ this theorem is due to Singh and Baloria [4].

(ii) For $p=2, q=1, x=0$ and $\gamma=1$ this theorem is due to Song and Yang [5].

Theorem 2. Let $f$ and $g$ be two entire functions of finite $(p, q)$-orders and $\lambda(f)>0$. Then, for $h>0$ and $p>q$,

$$
\lim _{r \rightarrow \infty} \frac{\left(\log ^{[p]} M(r, f o g)\right)^{1+x}}{\log ^{[p]} M\left(\exp ^{[p-1]}\left(\log ^{[q-1]} r\right)^{h}, f\right)}=0
$$

where $h>(1+x) \rho(g)$ if $p=2, q=1$ and $h>\rho(g)$ otherwise. Also $x \in(-\infty, \infty)$.

Proof. If $1+x \leq 0$ the theorem is trivial. So, we consider $1+x>0$. By the maximum modulus principle, we have

$$
M(r, f \circ g) \leq M(M(r, g), f),
$$

so that for all sufficiently large values of $r$,

$$
\begin{aligned}
\left\{\log ^{[p]} M(r, f o g)\right\}^{1+x} & <(\rho(f)+\varepsilon)^{1+x}\left\{\log ^{[q]} M(r, g)\right\}^{1+x} \\
& <(\rho(f)+\varepsilon)^{1+x}\left\{\exp ^{[p-q-1]}\left(\log ^{[q-1]} r\right)^{\rho(g)+\varepsilon}\right\}^{1+x}
\end{aligned}
$$

Again, for all sufficiently large values of $r$,

$$
\log ^{[p]} M(r, f)>(\lambda(f)-\varepsilon) \log ^{[q]} r .
$$

Hence, for all sufficiently large values of $r$,

$$
\frac{\left\{\log ^{[p]} M(r, f o g)\right\}^{1+x}}{\log ^{[p]} M\left\{\exp ^{[p-1]}\left(\log ^{q-1]} r\right)^{h}, f\right\}}<\frac{(\rho(f)+\varepsilon)^{1+x}\left\{\exp ^{[p-q-1]}\left(\log ^{[q-1]} r\right)^{\rho(g)+\varepsilon}\right\}^{1+x}}{(\lambda(f)-\varepsilon) \exp ^{[p-q-1]}\left(\log ^{[q-1]} r\right)^{h}}
$$

from which the theorem follows because we can choose $\varepsilon$ such that $0<\varepsilon<\min \left\{\lambda(f), \frac{h}{1+x}\right.$ $-\rho(g)\}$ if $p=2, q=1$ and $0<\varepsilon<\min (\lambda(f), h-\rho(g))$ otherwise.

Remark 2. If we take the condition $0<\rho(f)$ inplace of $\lambda(f)>0$ the theorem remains true with 'limit' replaced by 'limit inferior' and in this case improves and generalizes Theorem 2 of Singh and Baloria [4].

Theorem 3. Let $f$ and $g$ be two entire functions such that $0<\lambda(g) \leq \rho(g)<\infty$ and $\rho(f)<\infty$. Then, for $h>0$ and $p>q$,

$$
\lim _{r \rightarrow \infty} \frac{\left\{\log ^{[p]} M(r, f o g)\right\}^{1+x}}{\log ^{[p]} M\left(\exp ^{[p-1]}\left(\log ^{[q-1]} r\right)^{h}, g\right)}=0
$$

where $h>(1+x) \rho(g)$ if $p=2, q=1$ and $h>\rho(g)$ otherwise. Also $x \in(-\infty, \infty)$. 
We omit the proof of this theorem because it runs parallel to that of Theorem 2 .

Theorem 4. Let $f$ and $g$ be two entire functions of finite $(p, q)$-order with $\lambda(g)>$ $\rho(f) \geq \lambda(f)>0$. Then, for $p>q$,

$$
\lim _{r \rightarrow \infty} \frac{\log ^{[p]} M(r, \text { fog })}{\log ^{[p]} M\left(\exp ^{[p-1]}\left(\log ^{[q-1]} r\right)^{\rho(f)}, g\right)}=\infty .
$$

Proof. From (3.3), we have, for all $r \geq r_{0}$,

$$
\log ^{[p]} M(r, f o g)>(\lambda(f)-\varepsilon) \exp ^{[p-q-1]}\left(\log ^{[q-1]}\left(\frac{r}{2}\right)\right)^{\lambda(g)-\varepsilon}+(\lambda(f)-\varepsilon) o(1) .
$$

Also, for all $r \geq r_{0}$,

$$
\log ^{[p]} M(r, g)<(\rho(g)+\varepsilon) \log { }^{[q]} r .
$$

Taking $r$ so large that $\exp ^{[p-1]}\left(\log ^{[q-1]} r\right)^{\rho(f)} \geq r_{0}$, then

$$
\log ^{[p]} M\left(\exp ^{[p-1]}\left(\log ^{[q-1]} r\right)^{\rho(f)}, g\right)<(\rho(g)+\varepsilon) \exp ^{[p-q-1]}\left(\log ^{[q-1]} r\right)^{\rho(f)} .
$$

Thus, for sufficiently large $r$,

$$
\begin{aligned}
& \frac{\log ^{[p]} M(r, f o g)}{\log ^{[p]} M\left(\exp ^{[p-1]}\left(\log ^{[q-1]} r\right)^{\rho(f)}, g\right)} \\
> & \frac{(\lambda(f)-\varepsilon)) \exp ^{[p-q-1]}\left\{\log ^{[q-1]}\left(\frac{r}{2}\right)\right\}^{\lambda(g)-\varepsilon}+(\lambda(f)-\varepsilon) o(1)}{(\rho(g)+\varepsilon) \exp ^{[p-q-1]}\left(\log ^{[q-1]} r\right)^{\rho(f)}} .
\end{aligned}
$$

Since, $\lambda(g)>\rho(f)$ and we can choose $\varepsilon>0$ such that $\lambda(g)-\varepsilon>\rho(f)$. Thus,

$$
\lim _{r \rightarrow \infty} \frac{\log ^{[p]} M(r, f o g)}{\log ^{[p]} M\left(\exp ^{[p-1]}\left(\log ^{[q-1]} r\right)^{\rho(f)}, g\right)}=\infty
$$

and the theorem follows.

Remark 3. For $p=2$ and $q=1$ this theorem is due to Singh and Baloria [4].

We shall use the technique of the above theorems to get the parallel results on the maximum term of composition of entire functions. We prove:

Theorem 5. Let $f$ and $g$ be two entire functions such that $0<\lambda(f) \leq \rho(f)<\infty$ and $0<\lambda(g) \leq \rho(g)<\infty$. Then, for every positive constant $\gamma, p>q$ and every real number $x$,

$$
\lim _{r \rightarrow \infty} \frac{\log ^{[p]} \mu(r, f \circ g)}{\left\{\log ^{[p]} \mu\left(r^{\gamma}, f\right)\right\}^{1+x}}=\infty
$$


and

$$
\lim _{r \rightarrow \infty} \frac{\log ^{[p]} \mu(r, f o g)}{\left\{\log ^{[p]} \mu\left(r^{\gamma}, g\right)\right\}^{1+x}}=\infty
$$

Proof. If $x$ is such that $1+x \leq 0$, then the theorem is obvious. So, we suppose that $1+x>0$. Now, from (2.5) for all sufficiently large values of $r$,

$$
\mu(r, f \circ g) \geq \frac{1}{2} \mu\left(\frac{1}{16} \mu\left(\frac{r}{4}, g\right), f\right) .
$$

This gives,

$$
\log ^{[p]} \mu(r, f o g)>\frac{1}{2}(\lambda(f)-\varepsilon) \exp ^{[p-q-1]}\left(\log ^{[q-1]}\left(\frac{r}{4}\right)\right)^{\lambda(g)-\varepsilon}+\frac{1}{2}(\lambda(f)-\varepsilon) o(1)
$$

because in view of (2.1), we have for sufficiently large values of $r$,

$$
\mu(r, f)>\exp ^{[p-1]}\left(\log ^{[q-1]} r\right)^{\lambda(f)-\varepsilon} .
$$

Also, from (2.1) for all $r \geq r_{0}$,

$$
\log ^{[p]} \mu(r, f)<(\rho(f)+\varepsilon) \log ^{[q]} r
$$

and so for sufficiently large $r^{\gamma} \geq r_{0}$,

$$
\left\{\log ^{[p]} \mu\left(r^{\gamma}, f\right)\right\}^{1+x}<(\rho(f)+\varepsilon)^{1+x}\left(\log ^{[q]}\left(r^{\gamma}\right)\right)^{1+x} .
$$

Thus, as in Theorem 1, we find

$$
\lim _{r \rightarrow \infty} \frac{\log ^{[p]} \mu(r, f o g)}{\left\{\log ^{[p]} \mu\left(r^{\gamma}, f\right)\right\}^{1+x}}=\infty
$$

since we can choose $\varepsilon$ such that $0<\varepsilon<\min (\lambda(f), \lambda(g))$.

We omit the proof of (3.7).

Remark 4. The analogues to Theorem 2, Theorem 3 and Theorem 4 may later be filled by the reader.

Theorem 6. Let $f$ and $g$ be two entire functions of positive lower $(p, q)$-order and of finite $(p, q)$-order. Then, for every $h>0$,

$$
\lim _{r \rightarrow \infty} \sup \frac{\log ^{[p]} \mu\left\{4 \exp ^{[q-1]}\left(\frac{r^{1+h}}{4}\right), f o g\right\}}{\log ^{[p-1]} \mu\left(\exp ^{[q-1]} r, g\right)}=\infty .
$$

Proof. There exists a sequence $\left\{r_{n}\right\}, n=1,2, \ldots$ such that

$$
\mu\left(r_{n}, g\right)>\exp ^{[p-1]}\left(\log ^{[q-1]} r_{n}\right)^{\rho(g)-\varepsilon} .
$$


Let $R_{n}=\left(4 \log ^{[q-1]} r_{n}\right)^{1 /(1+h)}$, then

$$
\mu\left\{\exp ^{[q-1]}\left(\frac{R_{n}^{1+h}}{4}\right)\right\}>\exp ^{[p-1]}\left(\frac{R_{n}^{1+h}}{4}\right)^{\rho(g)-\varepsilon} .
$$

Now, from (2.1) and (2.5), for all $r \geq r_{0}$,

$$
\log ^{[p]} \mu(r, f \circ g)>\frac{1}{2}(\lambda(f)-\varepsilon) \log ^{[q]} \mu\left(\frac{r}{4}, g\right)+\frac{1}{2}(\lambda(f)-\varepsilon) o(1) .
$$

If $R_{n} \geq r_{0}$, then $4 \exp ^{[q-1]}\left(\frac{R_{n}^{1+h}}{4}\right) \geq r_{0}$ and so the above equation gives

$$
\begin{aligned}
& \log ^{[p]} \mu\left\{4 \exp ^{[q-1]}\left(\frac{R_{n}^{1+h}}{4}\right), f o g\right\} \\
> & \frac{1}{2}(\lambda(f)-\varepsilon) \log ^{[q]} \mu\left\{\exp ^{[q-1]}\left(\frac{R_{n}^{1+h}}{4}\right), g\right\}+\frac{1}{2}(\lambda(f)-\varepsilon) o(1) .
\end{aligned}
$$

Using (2.1), we find for the sequence $R_{n} \geq r_{0}$,

$$
\begin{aligned}
& \log ^{[p]} \mu\left\{4 \exp ^{[q-1]}\left(\frac{R_{n}^{1+h}}{4}\right), f \circ g\right\} \\
> & \frac{1}{2}(\lambda(f)-\varepsilon) \exp ^{[p-q-1]}\left(\frac{R_{n}^{1+h}}{4}\right)^{\rho(g)-\varepsilon}+\frac{1}{2}(\lambda(f)-\varepsilon) o(1) .
\end{aligned}
$$

Also, for all $r \geq r_{0}$,

$$
\log ^{[p-1]} \mu\left(\exp ^{[q-1]} r, g\right)<(r)^{\rho(g)+\varepsilon} .
$$

Thus, for the sequence $R_{n}\left(\geq r_{0}\right)$,

$$
\begin{aligned}
& \frac{\log ^{[p]} \mu\left\{4 \exp ^{[q-1]}\left(\frac{R_{n}^{1+h}}{4}\right), f o g\right\}}{\log ^{[p-1]} \mu\left(\exp ^{[q-1]} R_{n}, g\right)} \\
> & \frac{\frac{1}{2}(\lambda(f)-\varepsilon) \exp ^{[p-q-1]}\left(\frac{R_{n}^{1+h}}{4}\right)^{\rho(g)-\varepsilon}+\frac{1}{2}(\lambda(f)-\varepsilon) o(1)}{\left(R_{n}\right)^{\rho(g)+\varepsilon}} \\
\rightarrow & \infty \text { as } r \rightarrow \infty
\end{aligned}
$$

since we can choose $\varepsilon$ such that $0<\varepsilon<\min \left\{\lambda(f), \frac{h \rho(g)}{2+h}\right\}$.

Remark 5. For $p=2$ and $q=1$ this theorem is due to Singh and Baloria [4].

\section{References}

[1] J. Clunie, The composition of entire and meromorphic functions, Macintyre Memorial Volume, Ohio University Press, (1970), 75-92. 
[2] O. P. Juneja, G. P. Kapoor and S. K. Bajpai, On the $(p, q)$-order and lower $(p, q)$-order of entire function, J. reine angew. Math. 282(1976), 53-67.

[3] A. P. Singh, On the maximum term of composition of entire functions, Proc. Nat. Acad. Sci. India 59(A)I(1989), 103-115.

[4] A. P. Singh and M. S. Baloria, On the maximum modulus and maximum term of composition of entire functions, Indian J. pure appl. Math. 22(1991), 1019-1026.

[5] G. D. Song and C. C. Yang, Further growth properties of composition of entire and meromorphic functions, Indian J. pure appl. Math. 15(1984), 67-82.

Department of Mathematics, G. B. Pant University, Pantnagar-263145 (U. S. Nagar), Uttaranchal, India. 